\title{
Cerebellar syndrome with hydrocephalus due to Mycoplasma pneumoniae infection
}

\author{
R.J. Coleman, J.S. Brown, P. Butler and M. Swash \\ Departments of Neurology and Neuroradiology, The London Hospital, London E1 1BB, UK.
}

\begin{abstract}
Summary: A 27 year old woman developed a cerebellar syndrome with serological evidence of recent Mycoplasma pneumoniae infection. The cranial computed tomographic scan showed effacement of the fourth ventricle, enhancement of the basal meninges and hydrocephalus affecting the lateral and third ventricles. Clinical and radiological recovery occurred over 5 weeks. We propose that this was a manifestation of immune-mediated encephalomyelitis induced by the infection rather than direct invasion of the central nervous system.
\end{abstract}

\section{Introduction}

Mycoplasma pneumoniae infection is common in the United Kingdom, although its incidence is unknown. We describe a neurological complication of Mycoplasma pneumoniae infection and discuss its relationship to other reports of mycoplasma-related, post-infectious encephalomyelitis.

\section{Case report}

A 27 year old woman was admitted with a 2-day history of dry cough, vomiting, occipital headache and a feeling of unsteadiness. There was a history of chicken-pox three years previously. On examination, her pulse rate, blood pressure and temperature were normal and there was no neck stiffness or drowsiness. Cardiovascular, respiratory and abdominal examinations were normal. Visual fields, pupils and optic fundi were normal. There was a full range of pursuit eye movements but saccades were hypometric. There was marked cerebellar dysarthria with severe cerebellar ataxia of all four limbs and she was unable to walk without support. The tendon jerks were symmetrical and the plantar responses were both flexor.

The haemoglobin, white cell count, ESR and liver function tests were normal and serological tests for syphilis were negative. The chest radiograph showed miliary calcification consistent with previous chicken-pox infection. The visual evoked responses and electroencephalogram were normal. A cranial computed tomographic (CT) scan (Fig-

Correspondence: R.J. Coleman, B.Sc., M.D., M.R.C.P. Department of Neurology (Ward 8), Leeds General Infirmary, Great George Street, Leeds LS1 3EX, UK. Accepted: 16th February 1990 ure 1) showed swelling of the cerebellum and brain stem and there was abnormal enhancement of the basal meninges. The fourth ventricle was effaced and there was dilatation of the third and lateral ventricles, consistent with obstructive hydrocephalus. A lumbar puncture was carried out after prioro administration of intravenous mannitol. The cere brospinal fluid (CSF) was clear and colourless witho an opening pressure of $115 \mathrm{~mm} \mathrm{CSF}$; microscopy? showed 50 lymphocytes $/ \mathrm{mm}^{3}$. The CSF was sterile and the protein and glucose concentrations were normal. Isoelectric focusing revealed the presence of oligoclonal bands. The patient was initially treated with antituberculous chemotherapy but a weakly positive tuberculin test failed to support the diagnosis of tuberculous meningitis. A K veim test (biopsied at 6 weeks) showed no evidence of sarcoidosis. Using a latex agglutination test, serum antibodies against Mycoplasma pneumoniae were identified in a titre of 1 in 80; these antibodies were of the IgM class, indicating that the infection was recent. This result was not available until after clinical improvement had begun and specific antibiotic therapy was not prescribed. There was serological evidence of previous varicella infection but titres for other viruses were within the normal range.

Two weeks after its onset, the patient's ataxia began to improve. After a further 3 weeks there were no residual neurological deficits and the CT scan was normal.

\section{Discussion}

Neurological complications occur in $2 \%$ of reported infections with Mycoplasma pneumoniae. These neurological manifestations include enceph- 

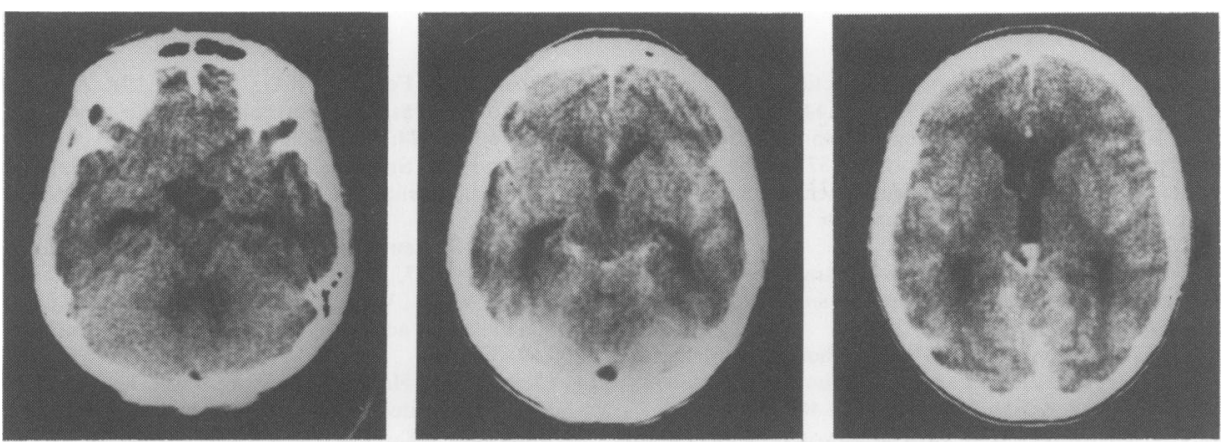

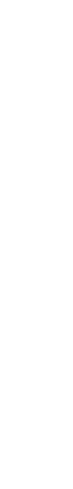

Figure 1 CT scan showing effacement of the fourth ventricle, enhancement of the basal meninges and hydrocephalus. (Left without contrast enhancement; centre and right with contrast enhancement.)

alitis, psychosis, hemiplegia, chorea, cerebellar ataxia, aseptic meningitis, cranial nerve palsies, transverse myelitis, polyradiculopathy and Guillain-Barré syndrome. ${ }^{2-10}$ Usually the neurological illness is self-limited and the prognosis is favourable.

In our patient the diagnosis of $M$. pneumoniae infection was based on serological detection of specific IgM antibodies; this diagnosis was suggested by the patient's dry cough although there was no clinical or radiological evidence of pneumonia. Infection with $M$. pneumoniae appears to have been responsible for the patient's cerebellar ataxia, which was associated with inflammatory changes in the basal meninges and a cellular response in the CSF. Hydrocephalus was due to compression of the fourth ventricle or aqueduct from swelling of the surrounding brain stem and cerebellum. In other reported cases, cerebellar ataxia occurred in association with evidence of diffuse cerebral involvement, ${ }^{3-5,9}$ but this was not apparent in our patient.

Cerebellar syndromes also occur in association with viral infections such as measles, mumps, chicken-pox and Epstein-Barr virus. ${ }^{11-14}$ As with $M$. pneumoniae infection, this is often part of a more generalized encephalopathy, sometimes associated with myelitis, inflammatory polyneuropathy or optic neuritis. In this syndrome of postinfectious encephalomyelitis the major pathological change is demyelination, but isolation of virus from CSF or brain tissue is rarely possible. Despite many common features, there are certain differences between the syndromes associated with individual organisms. For example, measles infection is characterized by diffuse cerebral involvement often followed by permanent neurological sequelae, whereas chicken-pox tends to be associated with a pure cerebellar syndrome and has a better prognosis. Post-infectious encephalomyelitis may be distinguished from acute viral encephalitis, which is primarily a disease of grey matter. ${ }^{15}$ In this condition there is direct invasion of the brain and it is often possible to isolate the causative organism (e.g. Herpes simplex, arboviruses) from CSF or from tissue samples. Some organisms are capable of producing more than one syndrome; for example, the mumps virus may be responsible for meningitis, encephalitis or post-infectious encephalomyelitis.

Post-infectious encephalomyelitis is probably immune-mediated. The evidence for this is: (i) there is a delay between the onset of the viral illness and its neurological complications; (ii) it is not usually possible to isolate virus from CSF or brain tissue; (iii) post-infectious encephalomyelitis shows clinical and pathological similarities with experimental allergic encephalitis in animals and with acute serum sickness in man. ${ }^{16}$ There is additional evidence that immune mechanisms are responsible for the encephalomyelitis that follows $M$. pneumoniae infection. For example, it is associated with other manifestations of immune-mediated disease, such as arthralgia, myositis, Guillain-Barré syndrome and glomerulonephritis, and in some cases circulating immune complexes have been isolated. ${ }^{10}$ Further, the time course of neurological improvement does not coincide with the timing of antibiotic therapy; ${ }^{9}$ in our case recovery occurred without specific antibiotic therapy. In clinical trials, steroid therapy has not been beneficial, ${ }^{17,18}$ despite anecdotal reports to the contrary.

In our patient a cerebellar syndrome occurred as a limited form of post-infectious encephalomyelitis, and the hydrocephalus was secondary to the cerebellar swelling. Mycoplasma antigen was not found in the CSF but the presence of oligoclonal bands suggests intrathecal synthesis of immunoglobulin, due either to tissue damage or in response to the infective agent. Prompt recognition of post-infectious encephalomyelitis is important since it allows inappropriate treatment to be avoided. 


\section{References}

1. Noah, N.D. Mycoplasma pneumoniae infection in the United Kingdom, 1967-73. Br Med J 1974, 2: 544-546.

2. Reiman, H.A. An acute infection of the respiratory tract with atypical pneumonia. JAMA 1938, 111: 2377-2384.

3. Skoldenberg, B. Aseptic meningitis and meningoencephalitis in cold agglutinin-positive infections. $\mathrm{Br}$ Med $J$ 1965, 1: $100-102$.

4. Hodges, G.R., Fass, R.J. \& Saslaw, S. Central nervous system disease associated with Mycoplasma pneumoniae infection. Arch Intern Med 1972, 130: 277-282.

5. Steele, J.C., Gladstone, R.M., Thanasophon, S. \& Fleming, P.C. Acute cerebellar ataxia and concomitant infection with M. pneumoniae. J Pediatr 1972, 80: 467-469.

6. Lerer, R.J. \& Kavalsky, S.M. Central nervous system disease associated with Mycoplasma pneumoniae infection. Pediatrics 1973, 52: 658-668.

7. Wyler, A.R. \& Harris, A.B. Cerebellar ataxia with $M y c o-$ plasma pneumoniae. Ann Intern Med 1974, 80: 556-557.

8. Fisher, R.S., Clark, A.W., Wolinsky, J.S., Parhad, I.M., Moses, H. \& Mardiney, M.R. Postinfectious leukoencephalitis complicating Mycoplasma pneumoniae infection. Arch Neurol 1983, 40: 109-113.

9. Hely, M.A., Williamson, P.M. \& Terenty, T.R. Neurological complications of Mycoplasma pneumoniae infection. Clin Exp Neurol 1984, 20: 153-160.
10. Behan, P.O., Feldman, R.G., Segerra, J.M. \& Draper, I.T. Neurological aspects of mycoplasmal infection. Acta Neurol Scand 1986, 74: 314-322.

11. Miller, H.G., Stanton, J.B. \& Gibbons, J.L. Para-infectious encephalomyelitis and related syndromes. $Q J$ Med 1956, 25: 427-505.

12. Tyler, H.R. Neurological complications of rubeola (measles). Medicine 1957, 36: 147-167.

13. Peters, A.C.B., Versteeg, J., Lindeman, J. \& Bots, G.T.A.M. Varicella and acute cerebellar ataxia. Arch Neurol 1978, 35: 769-771.

14. Cleary, T.G., Henle, W. \& Pickering, L.K. Acute cerebellar ataxia associated with Epstein-Barr virus infection. JAMA 1980, 243: 148-149.

15. Kennard, C. \& Swash, M. Acute viral encephalitis - its diagnosis and outcome. Brain 1981, 104: 129-148.

16. Johnson, R.T. The pathogenesis of acute viral encephalitis and post-infectious encephalomyelitis. J Infect Dis 1987, 155: 359-364.

17. Ziegra, S.R. Corticosteroid treatment for measles encephalitis. J Pediatr 1961, 59: 322-323.

18. Boe, J., Solberg, C.O. \& Saeter, T. Corticosteroid treatment for acute meningoencephalitis: a retrospective study of 346 cases. Br Med J 1965, 1: 1094-1095. 